\title{
Therapeutic Effect of Yiqi Tongnao Decoction on Acute Cerebral Infarction of Qi Deficiency and Blood Stasis Type
}

\author{
Jian Guo ${ }^{1, a}$, Deqi Ren²,b Xuehui Chang ${ }^{3, c}$ (corresponding author) \\ ${ }^{1}$ Henan Province Hospital of Traditional Chinese Medicine, Zhengzhou, Henan 450000 \\ ${ }^{2}$ Henan Province Hospital of Traditional Chinese Medicine, Zhengzhou, Henan 450000 \\ ${ }^{3}$ Henan University of Chinese Medicine, Zhengzhou, Henan 450000
}

\begin{abstract}
The object is to explore the clinical effect of applying Yiqi Tongnao Decoction in the treatment of patients with acute cerebral infarction of Qi deficiency and blood stasis. In this study, patients in our hospital were taken as the main research objects, all of whom were patients with acute cerebral infarction of Qi deficiency and blood stasis type. The number of patients is 100 . The study period is from January 2020 to January 2021. The 100 patients selected for this study are divided into two groups. One group is given conventional western medicine treatment as the control group, and the other group is given Yiqi Tongnao Decoction treatment as the experimental group, the therapeutic effects of the two groups of patients were compared and analyzed. The scores of various TCM syndromes of the experimental group were lower than those of the control group, and the scores of neurological deficit and blood viscosity after treatment were lower than those of the control group. The effective rate of treatment was $94.00 \%$, which was higher than $78.00 \%$ of the control group. The adverse reaction rate of patients in the group was $6.00 \%$, which was lower than that in the control group, and the differences in the data were statistically significant $(\mathrm{P}<0.05)$. The application of Yiqi Tongnao Decoction in patients with acute cerebral infarction of Qi deficiency and blood stasis type has significant clinical effects. It can improve the clinical symptoms of patients, reduce the incidence of adverse reactions, and has a positive significance in promoting the recovery of patients.
\end{abstract}

\section{Introduction}

Acute cerebral infarction has a high clinical incidence. The main factor leading to the patient's morbidity is insufficient blood supply to the patient's brain, which leads to the patient's atherosclerosis, the patient's local blood flow is blocked, and there is blockage, hypoxia and local tissue necrosis. And other issues, which posed a serious threat to the life and health of patients ${ }^{[1]}$. From the perspective of traditional Chinese medicine, acute cerebral infarction belongs to the category of stroke, and patients need to be treated according to syndrome differentiation ${ }^{[2]}$. This study mainly took patients with acute cerebral infarction of Qi deficiency and blood stasis type as an example, applying Yiqi Tongnao Decoction to explore its impact evaluation on the treatment effect of patients.

\section{Objective}

A comparative experiment was carried out to use conventional western medicine and Yiqi Tongnao Decoction to treat patients with acute cerebral infarction of Qi deficiency and blood stasis, and to compare their clinical treatment effects to confirm the application effect of Yiqi Tongnao Decoction in patients.

\section{Methods}

\subsection{General patient information}

The patients in our hospital were selected as the research objects. Among them, 100 patients with Qi deficiency and blood stasis were selected. The study was carried out in January 2020 and January 2021. In order to explore the clinical effects of different treatment methods, this study divided the patients into two groups --- an experimental group and a control group of 50 people in each group. In the experimental group, there were 32 males and 18 females. The maximum and minimum ages of the patients were 77 and 48 years old respectively. The average age of the patients was $(58.87 \pm 5.45)$. The course of the patients was 4-15 days. The average course of the disease was 415 days. It was $(8.44 \pm 1.22)$ days. In the control group, the number of male and female patients were 33 and 15 respectively. The oldest patient was 78 years old and the youngest was 49 years old. The average age of the patients was (59.34). \pm 5.45$)$ years old, the patient's course of disease is $5-15$ days, with an average course of $(8.22 \pm 1.49)$ days. The general data of the two groups of patients can be compared and meet the comparison standard.

Inclusion criteria: The patients are consistent with the 
diagnostic criteria of Qi deficiency and blood stasis syndrome in the "Guiding Principles for Clinical Research of New Chinese Medicines" ; the patients meet the diagnostic criteria for cerebral infarction in Western medicine, and are consistent with the diagnostic criteria for ischemic cerebrovascular disease in Neurology; the patients are in the acute phase; the patients are admitted to the hospital within $6 \mathrm{~h}-2$ weeks after the onset of the disease; the patients are informed about the study, and voluntarily participates in it, and signed an informed consent; the study was approved by the hospital ethics committee.

Exclusion criteria: patients with organ damage and blood system diseases; patients with mental stress; patients with transient cerebral ischemia; patients with intracranial hemorrhage under imaging examination;

\subsection{Research methods}

The treatment method of the control group is conventional western medicine. In the specific implementation process, Shuxuening injection is applied. The drug manufacturer is Shenwei Pharmaceutical Group Co., Ltd., the corresponding Chinese medicine standard is H150924, and the drug application dose is $20 \mathrm{ml}$, mix it with $250 \mathrm{ml}$ of normal saline, and intervene by intravenous drip, once a day.

Patients in the experimental group were treated with Yiqi Tongnao Decoction. In the specific treatment process, the main prescriptions were: Astragalus 60g, Salvia 30g, Dilong 12g, Chuanqiong 15g, and Caulis spatholobi $30 \mathrm{~g}$. The medicinal materials were soaked in water for 30 minutes. The method is to take $150 \mathrm{ml}$ medicinal solution each time, mix the three medicinal solutions, and take them three times a day, 30 minutes after meals. Both groups of patients were treated continuously for 2 weeks.

\subsection{Observation indicators}

TCM Syndrome Score: Apply the "Diagnosis and Curative Effect Criteria of TCM Symptoms", which mainly include shortness of breath, fatigue, hemiplegia, crooked mouth and eyes, heavy pulse, and white and greasy tongue coating. A high score indicates that the patient's symptoms are severe.

Therapeutic effect: It mainly includes three indicators: significant, effective and ineffective. Significant means the patient's symptoms are improved significantly, the patient returns to normal, and the symptom score is reduced by more than $70 \%$, and the patient's symptoms are improved. Patients in the experimental group were treated with Yiqi Tongnao Decoction. In the specific treatment process, the main prescriptions were: Astragalus $60 \mathrm{~g}$, Salvia 30g, Dilong 12g, Chuanqiong 15g, and Caulis spatholobi $30 \mathrm{~g}$. The medicinal materials were soaked in water for 30 minutes. Take $150 \mathrm{ml}$ medicinal solution each time, mix the three medicinal solutions, and take them three times a day, 30 minutes after meals. Both groups of patients were treated continuously for 2 weeks. Effective means the symptom score reduction is at $30 \%-70 \%$, ineffective means the patient has no significant change before and after treatment. Discharge inefficiency is the total effective rate of this study.

The degree of neurological impairment: The stroke scale is used to evaluate the neurological impairment before and after treatment. If the score is high, it means the patient's functional impairment is serious ${ }^{[3]}$.

Plasma viscosity: observe and record the plasma viscosity of the two groups of patients after treatment.

Incidence rate of adverse reactions: Observe the occurrence of adverse reactions of the two groups of patients, including bleeding, rash, nausea and vomiting, etc. Compare the incidence of the two groups of patients.

\subsection{Statistical methods}

Use the statistical software SPSS20.0 as a tool to perform statistical analysis on the data appearing in this study, verifying the comparison results of the measurement data $(\mathrm{x} \pm \mathrm{s})$ with the $\mathrm{t}$ value, and comparing the count data ( $\mathrm{n}, \%)$ The results were verified by the $\mathrm{X}^{2}$ value. When the results showed $\mathrm{P}<0.05$, it indicated that the differences between the groups had statistical analysis value.

\section{Result}

\subsection{Comparison of TCM Syndrome Points}

The experimental group's patients' shortness of breath, fatigue, hemiplegia, crooked mouth and eyes, thin pulse, and greasy tongue coating scores were lower than those of the control group. There was a big difference in the data between each group, $\mathrm{P}<0.05$, which was statistically significant.

Table1. Comparison of points of various TCM syndromes $(\mathrm{x} \pm \mathrm{s})$

\begin{tabular}{|c|c|c|c|c|c|}
\hline Group & $\begin{array}{l}\text { Shortness } \\
\text { of breath }\end{array}$ & Hemiplegia & $\begin{array}{c}\text { Crooked } \\
\text { mouth and } \\
\text { eyes }\end{array}$ & Thin pulse & $\begin{array}{l}\text { Greasy } \\
\text { tongue } \\
\text { coating }\end{array}$ \\
\hline $\begin{array}{l}\text { Experimental } \\
\text { group }(n=50)\end{array}$ & $1.09 \pm 0.44$ & $1.57 \pm 0.34$ & $1.32 \pm 0.14$ & $1.07 \pm 0.31$ & $0.77 \pm 0.32$ \\
\hline $\begin{array}{c}\text { Control group } \\
\qquad(\mathbf{n}=\mathbf{5 0})\end{array}$ & $3.98 \pm 1.03$ & $1.99 \pm 0.56$ & $2.45 \pm 0.75$ & $2.98 \pm 1.00$ & $2.83 \pm 0.85$ \\
\hline $\mathbf{t}$ & 4.304 & 7.664 & 6.586 & 5.405 & 4.235 \\
\hline $\mathbf{P}$ & $<0.05$ & $<0.05$ & $<0.05$ & $<0.05$ & $<0.05$ \\
\hline
\end{tabular}




\subsection{Therapeutic effect}

The effective rates of treatment for patients in the experimental group and control group were $94.00 \%$ and $78.00 \%$, respectively. The effective rates of the experimental group were higher, and the difference in data was statistically significant $(\mathrm{P}<0.05)$.

Table2. Comparison of effective treatment rates between the two groups of patients [n(\%)]

\begin{tabular}{ccccc}
\hline Group & Significant & Effective & Ineffective & Effective rate \\
\hline $\begin{array}{c}\text { Experimental } \\
\text { group }\end{array}$ & $32(64.00)$ & $15(30.00)$ & $3(6.00)$ & $47(94.00)$ \\
$\begin{array}{c}(\mathbf{n}=\mathbf{5 0}) \\
\begin{array}{c}\text { Control group } \\
(\mathbf{n}=\mathbf{5 0})\end{array}\end{array}$ & $20(40.00)$ & $19(38.00)$ & $11(22.00)$ & $39(78.00)$ \\
$\mathrm{X}^{2}$ & & & & 12.007 \\
$\mathrm{P}$ & & & & $<0.05$ \\
\hline
\end{tabular}

\subsection{Score of neurological deficit}

Before treatment, the difference in neurological deficit scores between the two groups of patients was small, $\mathrm{P}>0.05$. After treatment, the scores of the experimental group were significantly lower than those of the control group, and the data were statistically significant $(\mathrm{P}<0.05)$.

Table3. Scores of neurological deficits in the two groups before and after treatment $(\mathrm{x} \pm \mathrm{s})$

\begin{tabular}{ccc}
\hline Group & $\begin{array}{c}\text { Before } \\
\text { treatment }\end{array}$ & $\begin{array}{c}\text { After } \\
\text { treatment }\end{array}$ \\
\hline $\begin{array}{c}\text { Experimental } \\
\text { group } \\
(\mathbf{n = 5 0})\end{array}$ & $10.24 \pm 2.33$ & $5.11 \pm 1.24$ \\
$\begin{array}{c}\text { Control group } \\
(\mathbf{n}=\mathbf{5 0})\end{array}$ & $10.94 \pm 0.95$ & $7.89 \pm 2.38$ \\
$\mathbf{t}$ & 0.045 & 6.556 \\
$\mathbf{P}$ & $>0.05$ & $<0.05$ \\
\hline
\end{tabular}

\subsection{Blood viscosity}

Before treatment, the blood viscosity of the two groups of patients were relatively high, and there was no significant difference between the groups, $\mathrm{P}>0.05$. After treatment, the scores all decreased. In contrast, the experimental group scores dropped more, compared with the control group. The score formed a significant difference, $\mathrm{P}<0.05$, which was statistically significant.

Table4. Comparison of blood viscosity scores before and after treatment between the two groups $(\mathrm{x} \pm \mathrm{s})$

\begin{tabular}{ccc}
\hline Group & $\begin{array}{c}\text { Before } \\
\text { treatment }\end{array}$ & $\begin{array}{c}\text { After } \\
\text { treatment }\end{array}$ \\
\hline $\begin{array}{c}\text { Experimental } \\
\text { group } \\
(\mathbf{n}=\mathbf{5 0})\end{array}$ & $2.00 \pm 0.23$ & $1.21 \pm 0.05$ \\
$\begin{array}{c}\text { Control group } \\
(\mathbf{n}=\mathbf{5 0})\end{array}$ & $2.03 \pm 0.54$ & $1.89 \pm 0.33$ \\
$\mathbf{t}$ & 0.945 & 3.956 \\
$\mathbf{P}$ & $>0.05$ & $<0.05$ \\
\hline
\end{tabular}

\subsection{Adverse reaction rate}

The incidence of adverse reactions in the experimental group was $6.00 \%$, and the incidence of adverse reactions in the control group was $18.00 \%$. There was a significant difference in the comparative data, $\mathrm{P}<0.05$, which is meaningful.

Table5. Comparison of the incidence of adverse reactions between the two groups of patients [n(\%)]

\begin{tabular}{ccccc}
\hline Group & Bleeding & Rash & Sick and vomit & Incidence \\
\hline $\begin{array}{c}\text { Experimental } \\
\text { group (n=50) }\end{array}$ & $1(2.00)$ & $2(4.00)$ & $0(0.00)$ & $3(6.00)$ \\
$\begin{array}{c}\text { Control group } \\
(\mathbf{n}=\mathbf{5 0})\end{array}$ & $3(6.00)$ & $4(8.00)$ & $2(4.00)$ & $9(18.00)$ \\
$\mathrm{X}^{2}$ & & & 6.506 \\
$\mathrm{P}$ & & & $<0.05$ \\
\hline
\end{tabular}




\section{Conclusion}

Acute cerebral infarction is an acute ischemic cerebrovascular disease, which occurs mostly in the elderly, and most of the patients have underlying diseases $^{[4]}$. Patients usually have attacks when they are at rest and at rest. In the early stage of the onset, the rate of cerebral ischemia is relatively slow, and the patient can reach the peak in a few minutes. After the onset of the patient, hemiplegia, aphasia and other undesirable conditions are usually present. The clinical treatment of patients is usually by drug therapy, but the effect is limited ${ }^{[5]}$. From the perspective of traditional Chinese medicine, it believes that the patient belongs to the category of stroke, and the pathogenesis of ischemic stroke is mainly the deficiency of the brain and blood stasis. In the treatment of patients, it is necessary to replenish qi and promote blood circulation, remove blood stasis and dredge collaterals ${ }^{[6]}$. In this study, Yiqi Tongnao Decoction was mainly applied. Astragalus was the monarch medicine, which invigorated the vitality of the spleen and stomach. It can be used together with bloodactivating drugs to promote the smoothness of blood vessels and blood circulation, and restore brain nourishment. Earthworm is a ministerial medicine, which has the effects of activating collaterals, dysmenorrhea, removing blood stasis and promoting blood circulation. Danshen is an adjuvant, which has the functions of removing blood stasis and promoting blood circulation, relieving pain and regulating menstruation. Caulis spatholobi can nourish blood and promote blood circulation, sooth muscles and collaterals ${ }^{[7]}$. When all the medicines are used in combination, the effects of promoting blood circulation, nourishing qi, and clearing blood stasis can be realized, and the clinical application effect is remarkable.

In summary, the application of Yiqi Tongnao Decoction in the treatment of patients with acute cerebral infarction of Qi deficiency and blood stasis has significant clinical effects. It can improve the degree of neurological deficits and reduce the viscosity of blood pressure in patients. It has significant clinical application significance and should be promoted.

\section{Acknowledgement}

As this article is about to close, I would like to sincerely thank the people who helped me complete the thesis, thank you for your thoughts and guidance during the writing of this thesis, thank you for your careful guidance during the creation of the thesis. Secondly, I would like to thank the authors who consulted the relevant materials during the writing process of this thesis for providing reference materials for my thesis writing.

\section{Reference}

1. Lu Yongkun, Liu Xiangzhe, Yang Haiyan, et al. Effects of Buyang Huanwu Decoction on blood perfusion parameters in acute phase of cerebral infarction of Qi deficiency and blood stasis type[J]. Liaoning Journal of Traditional Chinese Medicine, 2019, 046(005): 1023-1026.

2. Chen Jia, Ke Shenghai, Zhang Xiuying, et al. Analysis of the clinical therapeutic value of Huangqi Jiaxuefuzhuyu decoction on cerebral infarction of Qi deficiency and blood stasis type[J]. Family Medicine, 2020, 000(003): 49.

3. Cheng Beibei, Liu Fang. Evaluation of the effect of Naogengtong Decoction on hemiplegia during recovery of cerebral infarction of Qi deficiency and blood stasis type[J]. Contemporary Medical Essays, 2020, v.18(01):198-199.

4. Wang Yan. Effect of Tongnao Restoring Decoction on Platelet Activation Indexes in Patients with Qi Deficiency and Blood Stasis Cerebral Infarction in the Recovery Period[J]. Journal of Practical Medical Techniques 2020, Volume 27, Issue 8, Pages 10941096, 2020.

5. Wu Hongqiang. Observation on the therapeutic effect of Yiqi Tongnao Decoction on acute cerebral infarction of Qi deficiency and blood stasis type[J]. Electronic Journal of Integrated Traditional Chinese and Western Medicine Cardiovascular Diseases, 2019, 007(034): 171.

6. Wang Haiying. Clinical study on the treatment of Qi deficiency and blood stasis type cerebral watershed infarction with the method of replenishing qi, nourishing blood and promoting blood circulation[D]. 2019.

7. Yang Xiaomei, Zhang Zhuanxia, Cai Xiaying. The clinical efficacy of Naogengtong Decoction in the treatment of hemiplegia during recovery of cerebral infarction of Qi deficiency and blood stasis type[J]. Liaoning Journal of Traditional Chinese Medicine, 2019, 046(002): 315-317. 